\title{
Ultraviolet Absorption Line Studies of Halo Gas
}

\author{
Klaas S. de Boer
}

Sternwarte, University of Bonn, Auf dem Hügel 71, D-53121 Bonn

\begin{abstract}
The status of halo studies using UV absorption lines is assessed. SirI absorption is essentially only seen from gas with $z \leq 1 \mathrm{kpc}$, and shows a modest flow toward the galactic disk. CIV shows diffuse absorption in general only from gas with $z \geq 1 \mathrm{kpc}$ with predominantly negative velocities. Three exceptional cases of gas showing CIV absorption without absorption by neutral components are discussed. Outflowing gas has not been detected thusfar.
\end{abstract}

\section{History}

The first mentioning of UV absorption by OVI, NV, and CIV in the literature occurs in 1956. Basing himself on knowledge of the discovery of 'high-velocity clouds' (HVCs) by Guido Münch at MtWilson, considerations of cloud stability led Spitzer (1956) to predict the existence of a galactic corona. He did not cite Münch's first publication on the subject (Münch 1952) but referred to 'Münch, in preparation', a paper which ultimately appeared as Münch \& Zirin (1961). For more details see de Boer (1989).

Spitzer then started to press for the realization of a space observatory. With the COPERNICUS (launched in 1973) and the Hubble Space Telescope (launched in 1990) he realised his early dream. In addition, the International Ultraviolet Explorer (IUE, launched 1978 and functioning until 1996), and more recently the Hopkins Ultraviolet Telescope (HUT), the Extreme Ultraviolet Explorer (EUVE), and the Orbiting Retrievable Far and Extreme Ultraviolet Spectrometer (ORFEUS) enabled to detect many features of the ultraviolet properties of interstellar gas in general and that of the local environment and the halo in particular.

Today we use not so much the word corona as Spitzer did, basing himself on the high temperatures of the solar corona, but instead the word halo. Halo derives from the Greek word for threshing ground. Threshing grounds are round. One can also imagine that light chaf blown into the air leads to roundish clouds lighting up in the sunlight (see de Boer 1989).

\section{Detectability, Metal Abundances}

Absorption line studies of halo gas have over the years been limited in their success mostly because of the lack of UV-bright background sources in the 
Milky Way halo. Stars available are the odd B-type stars suspected to be massive main-sequence like stars (but there is nagging doubt about the correctness of this typing; see for normal stars e.g. Keenan 1997) and the subdwarf stars of sdB, sdO, and pAGB type. Here the sdB stars are optimal being sufficiently hot to have clean spectra yet of still acceptable absolute magnitude (see e.g. de Boer et al. 1994).

For useful studies the stars have to be close to each other in the sky and at the same time be spread well in distance in order to sample the halo gas with distance. Furthermore, an extragalactic light source helps to verify the presence of absorbing agents in principle (van Woerden 1997). The most sensitive absorption lines for neutral clouds are those of C II at $1335 \AA$ and of MgII near $2800 \AA$ (for details see, e.g., de Boer 1997).

The gas in the halo of the Milky Way contains all the usual elements in abundance. This was demonstrated originally by Savage \& de Boer (1979) by the presence of absorbing HVCs (at +60 and $+120 \mathrm{~km} \mathrm{~s}^{-1}$ ) on the line of sight to the LMC containing C II, Fe II, MgII, SiII, and the like. Since then measurements in spectra of more distant extragalactic objects have corroborated the significant metal content of HVC gas (see review by Savage \& Sembach 1996). In fact, both for $\mathrm{Mg}$ and $\mathrm{Si}$ the abundance in halo gas lies, compared to galactic gas (see review by de Boer, Jura \& Shull 1987), in the galactic range of values. The highest element abundances quoted for diverse HVCs are $[\mathrm{Mg} / \mathrm{H}]>-0.59$ (Bowen et al. 1994) and $[\mathrm{Si} / \mathrm{H}]>-0.80$ (Danly, Albert \& Kuntz 1993). For the gas in the Magellanic Stream, which may be a mix of halo gas and Magellanic Cloud gas, these abundances are $[\mathrm{Mg} / \mathrm{H}]$ $>-0.49$ (Savage et al. 1993) and $[\mathrm{Si} / \mathrm{H}]>-0.70(\mathrm{Lu}$, Savage \& Sembach 1994).

\section{Neutral Diffuse Gas in the Halo}

The neutral diffuse halo gas manifests itself in the common absorption lines. The best studied lines are those of $\mathrm{Si}$ and $\mathrm{Mg}$, being numerous and among the strongest and thus allowing the determination of reliable column densities.

SiII lines have been investigated extensively, because the available ones allow to cover a large range in optical depth. The width of the SiII absorption toward halo stars is documented by Danly et al. (1992), reporting on their own and on archival IUE data. I have plotted that width in velocity units in Fig. 1, thereby selecting only data in the direction more or less toward the galactic pole. In this way one is not significantly hampered by effects of galactic rotation. The absorption range lies between approximately -60 and $+40 \mathrm{~km} \mathrm{~s}^{-1}$, the centroid of the absorption being near $-10 \mathrm{~km} \mathrm{~s}^{-1}$. The maximum and minimum velocity of absorption reported corresponds to $\tau \simeq 1$ in the Sill $1260 \AA$ line, which equals $N(\operatorname{Sill}) \simeq 2 \cdot 10^{12} \mathrm{~cm}^{-2}$, (or $N(\mathrm{H}) \simeq 6 \cdot 10^{16} \mathrm{~cm}^{-2}$ if solar abundance is assumed). Does the observed width of $100 \mathrm{~km} \mathrm{~s}^{-1}$ represent temperature? Or is it turbulent motion? Since 


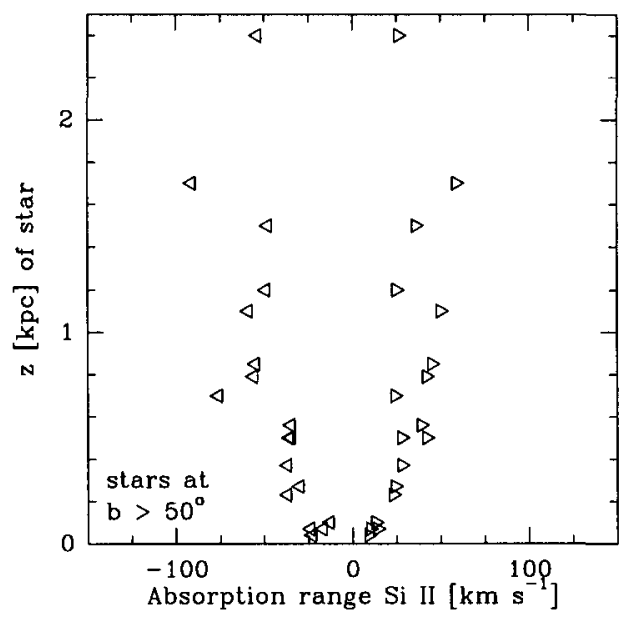

Fig. 1. The width of the interstellar absorption in Sill (triangles) is shown against the $z$-distance of the star used as background light source (see Sect. 3). The full width essentially stays constant for stars at $z>0.8 \mathrm{kpc}$ indicating no further absorption beyond that $z$-distance. The average velocity of absorption lies rather near $-10 \mathrm{~km} \mathrm{~s}^{-1}$, indicating a gentle downward motion. Absorption by Civ sets in not before $z=1 \mathrm{kpc}$ (see Sect. 5 )

the full width of absorption does not significantly increase beyond $z \sim 800$ pc, one may conclude that Sill exists predominantly within a small layer. The mean velocity together with the distance show that neutral gas of the lower halo on average descends slowly toward the Milky Way disk.

\section{Halo High Velocity Clouds}

The quest to find the distance to HVCs has been at last successful. Before I summarize the results I like to emphasize (again) that the formal distinction between HVCs and IVCs at $-100 \mathrm{~km} \mathrm{~s}^{-1}$ is artificial, since it does not take effects of galactic latitude and of galactic rotation into account. Yet, there are surely gas structures at 'intermediate' velocities at not too large $z$ distance, as well as true 'high-velocity' clouds. But equally sure there exist gas clouds at large $z$ having only small radial velocities, hiding in the absorption by local low velocity galactic gas.

Apart from the many lower limits (see for reviews with tabular material de Boer 1997 and Wakker \& van Woerden 1997) there are now the upper limits to the distance of $1.2 \leq z \leq 4.4 \mathrm{kpc}$ for gas between Complex M II and M III (Danly, Albert \& Kuntz 1993), of $z \leq 4.1 \mathrm{kpc}$ for gas at the edge of Complex $\mathrm{C}$ based on studies of stars in the direction to M 13 (de Boer \& 
Savage 1983; Bates et al. 1995; Shaw et al. 1996), of $z \leq 1.4 \mathrm{kpc}$ for Complex L (Albert et al. 1989), and of $25 \mathrm{kpc}$ for the HVC in the foreground of the LMC (Savage \& de Boer 1981; Molaro et al. 1993). Significant lower limits are $z>2.4 \mathrm{kpc}$ toward Cloud A in Complex C I (Schwarz, Wakker \& van Woerden 1995), and $z>3 \mathrm{kpc}$ toward Cloud A III (Wakker et al. 1996).

\section{Highly Ionized Gas}

Absorption by CrV normally shows up in spectra of stars only when $z>1$ kpc (see Danly et al. 1992). There the velocity width is larger than that of Sir at low $z$. Does this difference indicate a higher temperature? Or is it a width dominated by a real velocity spread? The latter is the more likely explanation because the absorption widths come from sight lines extending over a few kpc. Moreover, the pure temperature width of $\mathrm{Crv}$ absorption lines is $20 \mathrm{~km} \mathrm{~s}^{-1}$ for $2 \cdot 10^{5} \mathrm{~K}$, this temperature being the one of maximum abundance of $\mathrm{C}^{3+}$ in thermal equilibrium gas. If the gas were to be at $10^{6} \mathrm{~K}$, the thermal width of Crv absorption would be about $50 \mathrm{~km} \mathrm{~s}^{-1}$.

Furthermore, the CIV absorption line normally is smooth and does not show so much the component structure as the Sill lines do. Apparently the Sill absorption is more defined in velocity (in clouds?) whereas the CIV is rather due to diffuse material. However, temperature as well as the element's weight may contribute to the smoothness of the absorption.

$\mathrm{NV}$ is more difficult to detect than CIV , because the product of the Nv absorption line $f$-value and $N$ abundance is a factor of $\simeq 10$ smaller than that of CrV . First successes of NV measurements are by Savage \& Massa (1987), but one must note that their lines of sight run over substantial portions through the ever thicker disk in general galactocentric directions.

Collecting all the Crv and Nv data available to them, Savage \& Massa (1987) derived a scale height for these ions of $2-3$ and $1-4 \mathrm{kpc}$, respectively. This can be related to the scale height of free electrons of 1-2.5 kpc (Reynolds 1989).

OVI has been detected with ORFEUS in the spectra of several galactic stars at limited distances from the disk (Hurwitz \& Bowyer 1996), and toward an SMC star (Hurwitz et al. 1995). New high dispersion data have been taken Nov 1996 with ORFEUS but have not yet been analyzed in detail and thus nothing can be said about presence of OVI in relation to NV or CIV .

Dixon, Davidsen \& Ferguson (1996) give evidence from HUT spectra for OVI emission by halo gas. The most significant detection is in the direction $l=218.2^{\circ}, b=56.4^{\circ}$, a region devoid of high velocity gas.

Information on the distribution of highly ionized species in $z$ can be obtained from the structure of the absorption profile. When one adopts a model for the rotation of halo gas, and then translates the limits of each velocity bin into a distance bin, the column density derived from the absorption in that bin can so be transformed into a gas density for that distance bin. Doing 
so, Savage \& de Boer (1981; their Fig. 6) demonstrated that CIV may extend out to $z>10 \mathrm{kpc}$, based on the assumption that the halo gas corotates with the disk. We know now that this is not the case (see below). We now also know that CIV may exist at other temperatures than that for the peak appearance in thermodynamic equilibrium (see e.g. Böhringer, 1997), but even then CIV may still be present with a constant fraction of all $\mathrm{C}$ over a large temperature range. Thus the $n(z)$-profile of CIV as published by Savage \& de Boer (1981) most likely has to be modified only to a smaller $z$-extent due to slower rotation.

\section{Motion of Halo Gas}

From the survey of HVC detections at $21 \mathrm{~cm}$ by Giovanelli (1980) it is evident, that a substantial part of the observed velocity is the reflection of the galactic rotation of the Sun (see also Wakker 1991). However, more can be said.

In plots of $v_{\mathrm{HVC}}$ versus galactic longitude one finds that the sine-wave of the galactic rotation has at $l=180^{\circ}$ a velocity of $\simeq-100 \mathrm{~km} \mathrm{~s}^{-1}$. This means that the HVCs as a sample move at about that speed toward the rotation axis of the galaxy. Absorption lines toward the North Galactic Pole (star vZ 1128 in M 3) by de Boer \& Savage (1984) show a velocity of $\simeq-70$ $\mathrm{kms}^{-1}$, a motion toward the disk (see Fig. 1 for the slow flow of gas near the disk). Furthermore, on the sight line toward the globular cluster M 13 $\left(l=+60^{\circ}, b=+45^{\circ}\right)$ the absorption velocity is $\simeq-80 \mathrm{~km} \mathrm{~s}^{-1}$, although velocities of $0 \leq v \leq+20 \mathrm{kms}^{-1}$ are expected with corotation (de Boer \& Savage 1983). This means that in this direction the gas stays behind the disk rotation. Collectively these findings were used in a consistency test by Kaelble, de Boer \& Grewing (1985) and they concluded that, on average, halo HVC gas is at $z=3 \mathrm{kpc}$ and has velocities of up to $100 \mathrm{~km} \mathrm{~s}^{-1}$ toward the galactic rotation axis, of up to $100 \mathrm{~km} \mathrm{~s}^{-1}$ toward the galactic disk, and of only $100 \mathrm{~km} \mathrm{~s}^{-1}$ along with the disk rotation (see also de Boer, 1985).

\section{Three Cases of Absorption by C IV Without Si II or $\mathrm{H}_{\mathrm{I}}$}

In most of the absorption spectra CIV is present at the same velocity as SiII, albeit with a more smooth profile (see e.g. toward SN 1987A, Savage et al. 1989). There are now three cases known in which CIV is present without detection of Sili or of $\mathrm{HI}$.

Mrk 509. Sembach et al. (1995) found CIV at $-270 \mathrm{~km} \mathrm{~s}^{-1}$ in the spectrum of Mrk $509\left(l=36^{\circ}, b=-30^{\circ}\right)$, no SilI at that velocity, and $\mathrm{H}$ only at a position $2^{\circ}$ away. The velocity is exceptional; from galactic rotation one would expect velocities ranging from 0 to $+80 \mathrm{~km} \mathrm{~s}^{-1}$ for gas with $d<16$ 

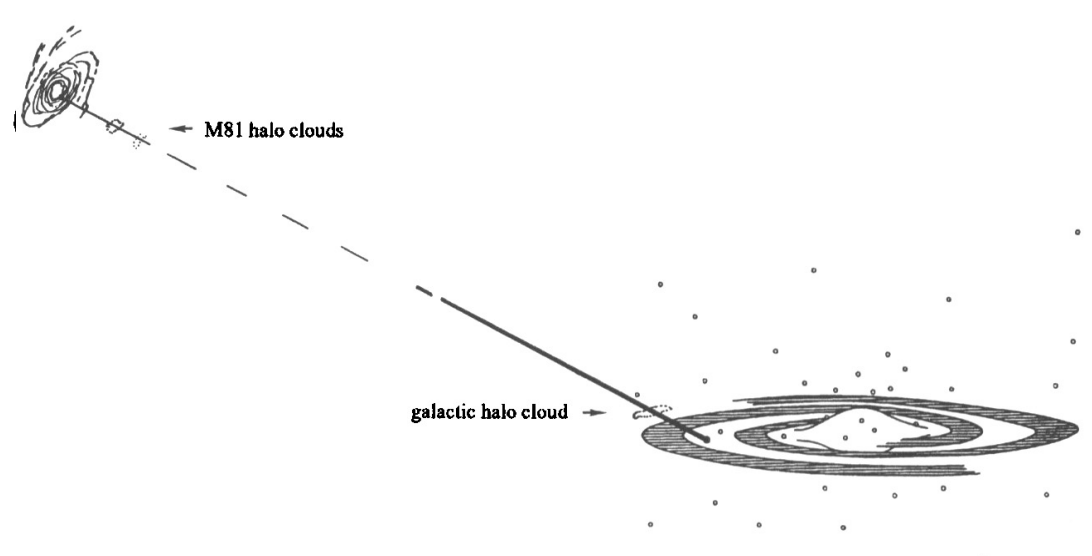

Fig. 2. The line of sight from the Milky Way to SN 1993J in M81 contains numerous clouds. Absorption near $0 \mathrm{~km} \mathrm{~s}^{-1}$ is due to gas in the solar vicinity, absorption near $-50 \mathrm{~km} \mathrm{~s}^{-1}$ due to a local halo cloud, absorption near $-120 \mathrm{~km} \mathrm{~s}^{-1}$ due to gas in M81. The absorption complex at velocities between +120 and $+240 \mathrm{~km} \mathrm{~s}^{-1}$ is due to clouds with very small spatial extent but with high column densities of $\mathrm{Ca}$ II, MgII , and CIV, somewhere along the $3 \mathrm{Mpc}$ line of sight, but most likely near M 81. For the exceptional absorption profiles see see Vladilo et al. (1993) and de Boer et al. (1993)

$\mathrm{kpc}$, or at most down to $-110 \mathrm{~km} \mathrm{~s}^{-1}$ at larger distances. However, corotation can only be an hypothesis so far away from the disk.

HD 119608. Toward stars inside galactic radio Loop I and IV Sembach, Savage \& Tripp (1997) detected CIV at $-45 \mathrm{~km} \mathrm{~s}^{-1} \quad\left(l=320^{\circ}, b=+43^{\circ}\right)$ without $\mathrm{Mg}$ II at $1239 \AA$. This gas most likely is part of the galaxy since its velocity is similar to that of other absorptions found in this general direction of the halo. However, a final check should be made by obtaining a spectrum of the much more sensitive line of $\mathrm{Mg}$ II at $2795 \AA$.

SN 1993J. On the sight line of $3.25 \mathrm{Mpc}$ toward SN 1993J in M $81(l=$ $142^{\circ}, b=+41^{\circ}$ ) absorption was found in CIV at the usual galactic velocities and at $\simeq-120 \mathrm{~km} \mathrm{~s}^{-1}$ (the velocity of M 81). In addition, strong absorption was found at $+130 \mathrm{~km} \mathrm{~s}^{-1}$ in Ca II, in Mg II, and in CIV (Vladilo et al. 1993, de Boer et al. 1993, Vladilo et al. 1994, Bowen, Blades \& Pettini 1994). Most extraordinary, the CIV absorption extended up to $200 \mathrm{~km} \mathrm{~s}^{-1}$, also in SiII, with the highest velocity cloud seen at $+240 \mathrm{~km} \mathrm{~s}^{-1}$. These velocities are not expected with any reasonable model for the dynamics of gas of the Milky Way. In addition, there is no trace of $\mathrm{H} \mathrm{I}$ in Effelsberg $21 \mathrm{~cm}$ spectra at these 


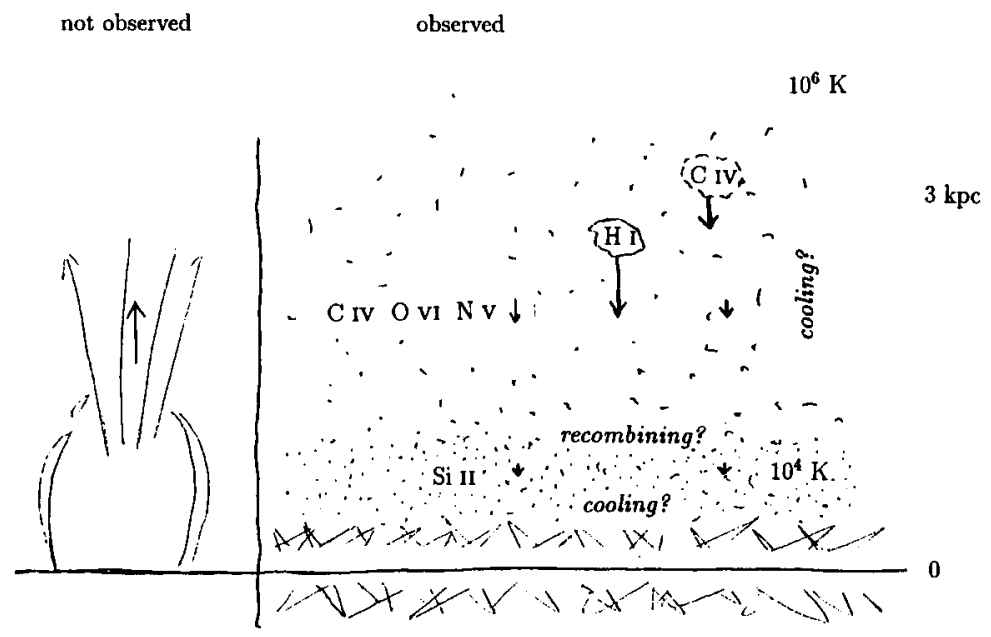

Fig. 3. The presence, the characteristics, and the flow of the material in the halo can be summarized as shown. Note that the sketch is a grand average but consistent with what is observed

velocities, implying that this gas can occupy only a very small fraction of the radio telescope beam, viz. that it is far away from us. The complex most likely represents HVCs falling toward M 81, or moving somewhere through intergalactic space (see Fig. 2).

\section{Outflow of Gas?}

The SiII absorption lines detected in spectra of halo stars at high galactic latitudes show a certain width in velocity. Taking these velocities at face value one might conclude that there is gas flowing away from as well as toward the disk. On the other hand, normal turbulence of clouds may account for a substantial fraction of the width. In the CIV absorption lines the width is mostly toward negative velocities. There is, to my knowledge, no detection of positive velocity CIV, except of course those structures which readily can be explained as due to normal galactic rotation (such as the CIV toward LMC stars).

Positive velocity clouds were detected toward M 81. These can, however, be anywhere on the line of sight and the more likely explanation is that they are high velocity clouds descending in the M 81 halo. 
My conclusion is, therefore, that there is as yet no evidence from observations for outflow. This means only that outflow is not seen in the absorption lines used thusfar. If very hot gas is flowing out, the OVI absorption lines may the first to give evidence.

\section{Conclusions}

Material in the halo at low $z$ is predominantly neutral, further out it is ionized. Temperatures are uncertain. High- and intermediate velocity clouds come down toward the disk. Outflowing halo material has not been seen in observational data. Most likely the gas at larger $z$ rotates along with the disk but at a slower pace. A sketch summarizing these views is given in Fig. 3 .

\section{References}

Albert C.E., Blades J.C., Morton D.C., Proulx M., Lockman F.J., 1989, in IAU Coll. 120, 'Structure and Dynamics of the Interstellar Medium', eds G. Tenorio-Tagle et al., Springer Lect. Notes in Physics No 350, p. 442

Bates B., Shaw C.R., Kemp S.N., Keenan F.P., Davies R.D., 1995, ApJ 444, 672

Böhringer H., 1997, these proceedings

Bowen D.V., Roth K.C., Blades J.C., Meyer D.M., 1994, ApJ 420, L 71

Bowen D., Blades J.C., Pettini M., 1995, ApJ 448, 662

Danly L., Albert C.E., Kuntz K.D., 1993, ApJ 416, L 29

Danly L., Lockman F.J., Meade M.R., Savage B.D., 1992, ApJS 81, 125

de Boer K.S., 1985, Mitteilungen Astron. Ges. 63, 25

de Boer K.S., 1989, in IAU. Coll. 120, 'Structure and Dynamics of the Interstellar

Medium', eds. G. Tenorio-Tagle et al.; Springer Lect. Notes in Physics No 350, p. 432

de Boer K.S., 1997, in 'The Physics of Galactic Halos', eds H. Lesch et al., Akademie Verlag, Berlin, p. 89

de Boer K.S., Savage B.D., 1983, ApJ 265, 210

de Boer K.S., Savage B.D., 1984, A\&A 136, L 7

de Boer K.S., Jura M.A., Shull J.M., 1987, in 'Exploring the Universe at Ultraviolet

Wavelengths', eds Y. Kondo et al.; Dordrecht, Reidel; p. 485

de Boer K.S., Rodriguez Pascal P., Wamsteker W., Sonneborn G., Fransson C., Bomans D.J., Kirshner R.P., 1993, A\&A 280, L 15

de Boer K.S., Altan A.Z., Bomans D.J., Lilienthal D., Moehler S., van Woerden

H., Wakker B.P., Bregman J.N., 1994, A\&A 286, 925

Dixon W.V.D., Davidsen A.F., Ferguson H.C., 1996, ApJ 465, 288

Giovanelli R., 1980, AJ 85, 1155

Hurwitz M., Bowyer S., Kudritzki R.-P., Lennon D.J., 1995, ApJ 450, 149

Hurwitz M., Bowyer S., 1996, ApJ 465, 296

Kaelble A., de Boer K.S., Grewing M., 1985, A\&A 143, 408

Keenan F., 1997, in 'Third Conference on Faint Blue Stars', eds A.G.D. Philip et al., Davis Press, Schenectady, in press

Lu L., Savage B.D., Sembach K.R., 1994, ApJ 437, L 119 
Molaro P., Vladilo G., Monai S., D'Odorico S., Ferlet R., Vidal-Madjar A., Dennefeld M., 1993, A\&A 274, 505

Münch G., 1952, PASP 64, 312

Münch G., Zirin H., 1961, ApJ 133, 11

Reynolds R.J., 1989, ApJ 339, L 29

Savage B.D., de Boer K.S., 1979, ApJ 230, L 77

Savage B.D., de Boer K.S., 1981, ApJ 243, 460

Savage B.D., Massa D., 1987, ApJ 314, 380

Savage B.D., Sembach K.R., 1996, ARAA 34, 279

Savage B.D., Jenkins E.B., Joseph C.L., de Boer K.S., 1989, ApJ 345, 393

Savage B.D., Lu L., Bahcall J.N., Bergeron J., Boksenberg A., et al., 1993, ApJ 413,116

Schwarz U.J., Wakker B.P., van Woerden H., 1995, A\&A 302, 364

Sembach K.R., Savage B.D., Trip T.M., 1997, ApJ 480, 216

Sembach K.R., Savage B.D., Lu L., Murphy E.M., 1995, ApJ 451, 616

Shaw C.R., Bates B., Kemp S.N., Keenan F.P., Davies R.D., Roger R.S., 1996, ApJ 473,849

Spitzer L., 1956, ApJ 124, 20

van Woerden H., 1997, these proceedings

Vladilo G., Centurión M., de Boer K.S., King D.L., Lipman K., Stegert J., Unger S.W., Walton N., 1993, A\&A 280, L 11

Vladilo G., Centurión M., de Boer K.S., King D.L., Lipman K., Stegert J., Unger S.W., Walton N., 1994, A\&A 291, 425

Wakker B.P., 1991, A\&A 250, 499

Wakker B.P., van Woerden H., 1997, ARAA 35, 217

Wakker B., Howk C., Schwarz U., van Woerden H., Beers T., Wilhelm R., Kalberla P., Danly L., 1996, ApJ 473, 834 